\title{
ATMA: Advertisement-based TDMA Protocol for Bursty Traffic in Wireless Sensor Networks
}

\author{
Surjya Ray, Ilker Demirkol and Wendi Heinzelman \\ Department of Electrical and Computer Engineering \\ University of Rochester, Rochester, New York \\ Email: $\{$ ray, demirkol, wheinzel\}@ece.rochester.edu
}

\begin{abstract}
Energy conservation is one of the primary goals of the majority of MAC protocols designed for wireless sensor networks. In Sensor-MAC (S-MAC), one of the first MAC protocols designed for sensor networks, nodes sleep and wake up periodically to save energy. MAC protocols such as Timeout-MAC (T-MAC) and Advertisement-MAC (ADV-MAC) improved upon S-MAC by introducing support for variable loads and providing further energy savings. In event-driven networks with sources generating bursty traffic or networks with period traffic, we can further reduce the energy consumption and increase node lifetime if we take into account the nature of the traffic. In this paper we propose Advertisement-based Time-division Multiple Access, or ATMA, a distributed TDMA-based MAC protocol for wireless sensor networks that utilizes the bursty or periodic nature of the traffic to prevent energy waste through advertisements and reservations for data slots. We provide detailed comparisons of the ATMA protocol with S-MAC, T-MAC and ADV-MAC through extensive simulations. The simulation results show that in bursty or periodic traffics, ATMA outperforms S-MAC, T-MAC and ADV-MAC in terms of energy consumption with reductions of up to $75 \%$, while faring as well as T-MAC and ADV-MAC in terms of packet delivery ratio and latency.
\end{abstract}

\section{INTRODUCTION}

Sensor nodes in wireless sensor networks are mostly battery powered devices that are designed to operate unattended for long periods of time. In order to maximize network lifetime, energy conservation and management is one of the primary goals of the majority of the MAC protocols designed for wireless sensor networks. The major cause of energy waste in conventional MAC protocols is idle listening. Nodes in MAC protocols such as IEEE 802.11 [1] listen to the channel continuously, because they do not know when they might receive a message. Measurements have shown that energy consumed in idle listening is comparable to that consumed in receiving or transmitting. For example, the receive:send:idle power ratios on MicaZ motes are 1.13:1:1.13 [2], while the same ratios for Tmote Sky motes are 1.11:1:1.11 [3]. In many sensor networks, messages are sent in bursts when an event is sensed. At all other times, most nodes have no data to send. If a conventional MAC protocol is used in sensor networks, a very large part of the energy will be wasted in idle listening.

This work was supported in part by NSF CAREER grant \#CNS-0448046 and in part by a Young Investigator grant from the Office of Naval Research, \#N00014-05-1-0626.
Most of the MAC protocols designed for sensor networks aim to minimize this energy wasted in idle listening.

This paper presents Advertisement-based Time-division Multiple Access, or ATMA, a distributed TDMA-based MAC protocol designed to minimize the energy wasted in idle listening and to take the nature of the traffic into account while contending for the medium. ATMA reduces energy waste by utilizing a combined advertisement and reservation scheme. Simulations show that ATMA performs very well in bursty traffic, with substantial energy savings as high as $75 \%$ compared to other well-known WSN MAC protocols, Sensor-MAC (S-MAC) [4], Timeout-MAC (T-MAC) [5] as well as compared to a CSMA-based protocol shown to present energy improvements on these protocols, namely ADV-MAC [6]. ATMA achieves these energy savings without degrading the performance in terms of latency and packet delivery ratio.

The rest of the paper is organized as follows. In Section II, we present a brief survey of existing energy-conserving MAC protocols. In Section III, we present the design of the ATMA protocol. In Section IV, we describe our simulation setup, followed by a detailed comparison of ATMA with SMAC, T-MAC and ADV-MAC using extensive simulations. Finally in Section V, we conclude the paper.

\section{RELATED WORK}

MAC protocols proposed for wireless sensor networks can be categorized as TDMA-based MAC protocols and contention-based MAC protocols. TDMA protocols are based on reservation and scheduling [7], [8], [9]. Each frame is divided into slots, and collision free communication is guaranteed by scheduling unique slots for each communicating node. TDMA-based protocols are very energy efficient when the network is highly loaded and all slots are used. The main challenges of TDMA-based protocols are synchronization issues and determining collision free schedules in a multi-hop networks. The nodes in TDMA-based protocols need to be synchronized, forming communication clusters. It is not trivial to maintain clock synchronization among the nodes, especially in a large network. Also, when new nodes join the network or nodes leave the network, it is not easy to dynamically change the frame length and slot assignment in a cluster. Furthermore, developing an efficient collision-free schedule with a high degree of channel reuse is difficult, and the solution is NP-hard 
[10]. Examples of TDMA-based MAC protocols are L-MAC [8], SS-TDMA [11], PACT [12], NAMA [9] and TRAMA [7]. SS-TDMA is applicable only to grid-like topologies, L-MAC and NAMA do not consider energy constraints of the sensors, and PACT requires a backbone network. ATMA improves upon these existing techniques as it is topology independent, takes energy conservation into account and is decentralized.

TRAMA [7] is an energy-conserving, distributed slot selection algorithm, similar to ATMA. However, to guarantee a packet delivery ratio (PDR) of $99 \%$, the number of signal packet retransmissions is set to 7 in TRAMA. This results in much longer delay and higher energy consumption compared with $10 \%$ S-MAC. ATMA, on the other hand uses a simple ACK for advertisement packets, and the proper choice of the advertisement (ADV) period duration achieves the same PDR as TRAMA while at the same time achieving delays and energy consumption values close to or smaller than $10 \% \mathrm{~S}$ MAC.

Contention-based protocols are widely employed because of their simplicity, robustness and flexibility. These protocols require little clock synchronization and no global topology information. Examples of such protocols are S-MAC [4], TMAC [5] and ADV-MAC [6]. These MAC protocols introduce a sleep-listen cycle into contention-based protocols to save energy wasted in idle listening. The nodes in such protocols go to a low power sleep state whenever possible to save energy.

The basic idea of S-MAC is to divide time into frames. Every frame has an active and a sleeping part. During the active part, nodes can communicate with their neighbors. The nodes turn off their radios and sleep to save energy during the sleeping part. The duty cycle, i.e., the proportion of active time within a frame, is set based on factors such as network density and message rate. Its value is decided before the network is set up and is static. Also, only one node can transmit in each frame in a neighborhood.

However, S-MAC is not suitable for variable traffic loads because of its static duty cycle. T-MAC [5] was proposed to improve the poor performance of S-MAC under variable loads. In T-MAC, the active period ends when no activation event, such as sensing of any communication on the radio, has occurred in the channel for a time threshold TA. Nodes will keep on renewing their timeout values whenever an activation event occurs. When none of these events occur for a duration of a timeout period, the nodes go to sleep.

The TA timeouts make the active period in T-MAC adaptive to variable traffic loads. However, whenever an activation event occurs, all nodes that hear the event renew their TA timer even if they are not a part of the transmission. As a result, nodes still end up wasting valuable energy. ADV-MAC [6] prevents this energy waste by using an advertisement period instead of a timeout period to alert intended receivers of packets waiting for the data contention. Nodes that are not part of the following data exchange go to sleep, saving energy.

In ATMA, we eliminate the two phase contention of ADVMAC by converting it to a slotted TDMA protocol. Nodes having data to send use the advertisement period to advertise for packets and reserve data slots. In the data period, packets are sent directly. Simulation results shows that ATMA outperforms ADV-MAC, T-MAC and S-MAC in networks with bursty and periodic traffic with substantial energy savings.

There are other MAC protocols that employ advertisement or reservation such as MH-TRACE (Multihop Time Reservation using Adaptive Control for Energy efficiency) [13]. MHTRACE organizes the network into one-hop clusters controlled by cluster-heads. Compared to MH-TRACE, we present a protocol for decentralized, clusterless networks.

\section{ATMA DESIGN OVERVIEW}

Time in ATMA is divided into frames. Each frame begins with a SYNC period, followed by an advertisement period and ending with a data period. The SYNC period is used for loose synchronization between nodes. The advertisement period is contention-based, and used for advertising for data and for reserving data slots. The data period is divided into slots for data exchange and is accessed in a contention-free manner.

The method of synchronization is the same as in S-MAC [4]. A frame is on the order of a second, which is $10^{4}$ times normal clock drifts. The SYNC and advertisement periods are contention-based, and, as such, these small drifts do not prevent the exchange of packets. To minimize the effect of these clock drifts in the contention-free data period, we set the data slots to be slightly larger than the duration of a data and an ACK packet. Also, the sending nodes begin transmitting after a small offset. Thus, small clock drifts do not affect the synchronization.

\section{A. Advertisement Period}

The advertisement (ADV) period is divided into many small slots, the size of which depends on clock resolution. The size of each ADV packet is a multiple of the slot duration. Each ADV packet contains the ID of the receiver and the chosen data slot number of the data period. All nodes are awake during this period. Each node having data to send randomly selects a slot at the beginning of the ADV period and initializes a timer to that slot value. When the timer reaches zero, the node transmits an ADV packet and waits for an acknowledgement from the intended receiver. If the intended receiver receives the ADV packet successfully, it replies with an advertisementacknowledgement packet, or A-ACK, that contains its own ID and the data slot number. Successful transmission of an ADV and its A-ACK will ensure that all nodes in the two hop neighborhood are aware of which data slots are being used. Hence, different senders will choose unique slots, preventing collisions in the data period. If an intended receiver node receives an ADV packet and knows that the selected slot is already occupied by another node, it does not send an A-ACK.

If a node that has data to send and is waiting to transmit its ADV hears another transmission, it will freeze its timer and wait for the entire transmission (ADV and A-ACK) to be over. Then, it will resume its timer again and transmit its ADV packet when the timer expires if there is time left in the ADV period for its ADV packet transmission. 
Initial Slot Selection:

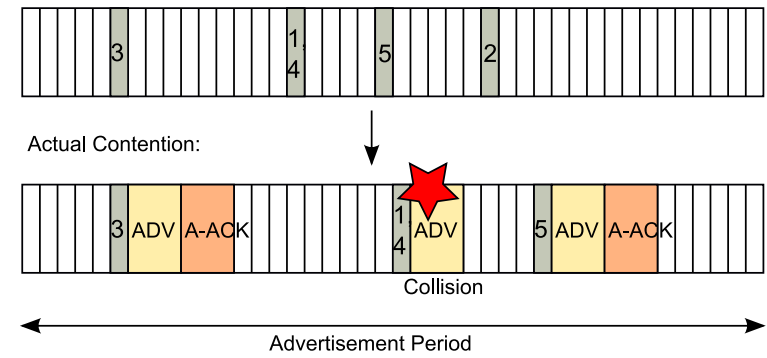

Fig. 1. Examples of the operation of the advertisement period. The numbers in the slots indicate the node IDs of nodes choosing those slots.

Fig. 1 shows an example of the operation of the ADV period. In the example, nodes with index numbers from 1 to 5 select random slots and initialize their timers. Node 3 selects slot 6 , nodes 1 and 4 both selects slot 16 , and so on. When the timer of node 3 reaches zero, it transmits its ADV packet with the ID of the intended receiver and the chosen data slot number. Other nodes will hear this transmission and will freeze their timers. Since no nodes chose slot 6 other than node 3, its transmission will be a success, and it will receive back an A-ACK from its intended receiver. All nodes will resume their timers after the end of the A-ACK transmission. Nodes 1 and 4 chose the same slot and hence their ADV packets possibly collide. However, if both receivers are out of the other transmitter's range, no collision will occur and both nodes will receive an A-ACK for their reserved slots. If a collision happens at an intended receiver, no A-ACK will be received by the corresponding transmitter. After these transmissions, node 5 will transmit successfully, but node 2 will have no time left in the ADV period to complete its transmission, and thus it will postpone its transmission to the next frame.

We can take advantage of bursty traffic to reduce the overhead in the ADV period and hence reduce the size of the ADV period to save energy. When a node needs to transmit packets in a burst, one single ADV/A-ACK exchange may reserve the same data slot for $n$ consecutive frames, so that the transmitting node need not send an ADV for each data packet. This can greatly reduce the traffic in the ADV period, saving energy. The number of consecutive frames for which a data slot is reserved may be fixed or may be announced in the ADV and A-ACK packets. In this paper, we consider a fixed value of $n$. Nodes may have fewer than $n$ packets. In that case, the receiver will timeout and go to sleep in the selected data slot. This technique also works with periodic traffic, with a period matched to the frame length, where a single ADV/A-ACK exchange can reserve a particular data slot, and the transmitter node can renew its channel access periodically.

\section{B. Data Period}

The data period is divided into longer slots compared to the slots of the ADV period. Each data slot is large enough to transmit a data packet along with an ACK. If a transmitting and a receiving node exchange ADV and A-ACK packets
TABLE I

PARAMETER VALUES

\begin{tabular}{c||c}
\hline \multicolumn{1}{c||}{ Parameters } & Values \\
\hline \hline Duration of ADV period of ADV-MAC & $15 \mathrm{~ms}$ \\
\hline Duration of ADV period of ATMA & $5 \mathrm{~ms}$ \\
\hline Duration of frames & $236.4 \mathrm{~ms}$ \\
\hline Duration of one ADV slot & $0.1 \mathrm{~ms}$ \\
\hline Duration of data slots of ATMA & $12 \mathrm{~ms}$ \\
\hline Duration of sync period & $8.4 \mathrm{~ms}$ \\
\hline Duration of control packet & $0.9 \mathrm{~ms}$ \\
\hline Duration of data packet & $8.5 \mathrm{~ms}$ \\
\hline Rx/Idle listening power & $59.1 \mathrm{~mW}$ \\
\hline Tx power & $52.2 \mathrm{~mW}$ \\
\hline Total simulation time & $200 \mathrm{~s}$ \\
\hline Transmission rate & $250 \mathrm{Kbps}$ \\
\hline Transmission range & $100 \mathrm{~m}$ \\
\hline Carrier sense range & $200 \mathrm{~m}$ \\
\hline
\end{tabular}

successfully, they will wake up at the beginning of their chosen slot to exchange data. In all other slots they will be asleep. Nodes that do not have any data to send or receive will be asleep during the entire data period.

New nodes joining the medium will initially have no information about the available data slots. If such a node has data to send, it can stay awake in the data period to find an unoccupied slot. It may attempt to reserve that slot in the next frame. If, however, the intended receiver knows that this slot is already reserved by another node (not in the transmitter's range), the intended receiver will simply not reply to the ADV packet.

\section{EXPERIMENTS}

In our experiments, we compared the performance of ATMA with three other protocols: S-MAC, T-MAC and ADV-MAC. We used energy consumption, throughput and latency as the three performance metrics for comparison.

\section{A. Simulation Setup}

We performed all simulations in ns 2.29 [14]. The S-MAC code is included in this version of ns. We coded T-MAC, ADV-MAC and ATMA in ns-2 as well. We use different duty cycle settings for S-MAC, specifically duty cycles of $10 \%$ and $20 \%$, because one fixed duty cycle is not suitable for all traffic loads investigated. The frame time for $10 \%$ duty cycle of S-MAC is $236.4 \mathrm{~ms}$, and we set this frame time for ATMA, T-MAC and ADV-MAC as well. The transmission rate is $250 \mathrm{kbps}$, and the transmission range is $100 \mathrm{~m}$, while the interference and carrier sense range is $200 \mathrm{~m}$. We use the power consumption values of MicaZ motes [2] to derive realistic energy consumption values. The current consumption values of MicaZ motes are $17.4 \mathrm{~mA}$ and $19.1 \mathrm{~mA}$ for transmission and reception, respectively. Considering a battery of $3 \mathrm{~V}$, the transmission and reception powers are $52.2 \mathrm{~mW}$ and $59.1 \mathrm{~mW}$, respectively. The idle power consumption is the same as that for reception. The ADV period and the data contention period are divided into slots of $0.1 \mathrm{~ms}$ each. For the multihop simulations, the nodes are deployed in the target area 


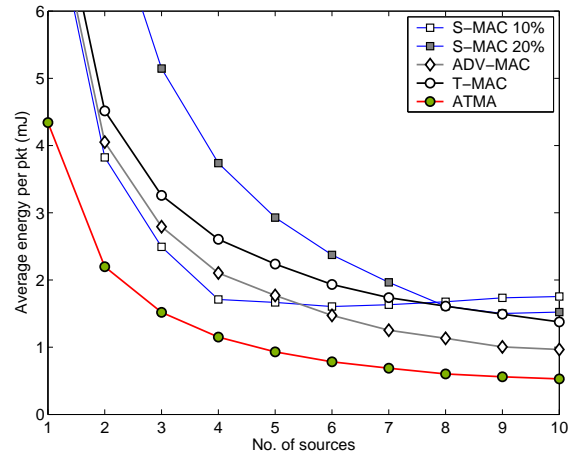

(a) Energy Consumption vs. No. of Sources.

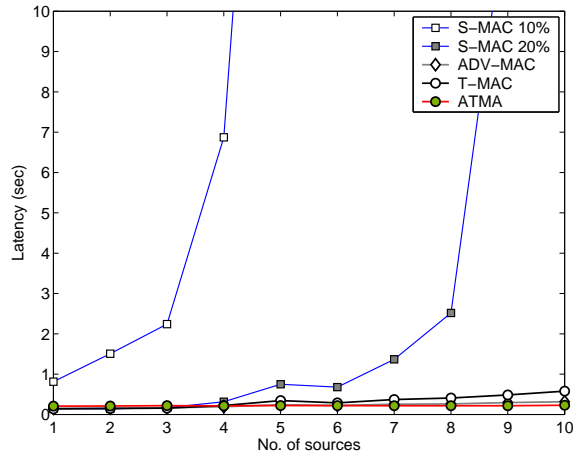

(b) Latency vs. No. of Sources.

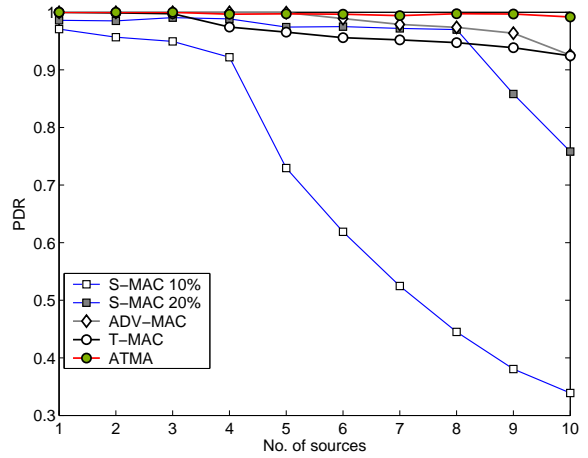

(c) PDR vs. No. of Sources.

Fig. 2. Single hop scenario. Performance as a function of the number of sources for ATMA, ADV-MAC, T-MAC and S-MAC.

with uniformly random distribution. We use T-MAC with overhearing avoidance, since it is used in the simulations in [5]. The simulation time is $200 s$, and each point in the figures is the average of 50 runs. The values used for the simulation parameters are summarized in Table I.

1) Effect of number of sources, single hop scenario: In the first set of simulations, we investigate the effect of the number of sources on the performance of the MAC protocols. We consider 20 nodes in an area of $50 \mathrm{~m} \times 50 \mathrm{~m}$ with all nodes being in the transmission range of each other, and we vary the number of sources from 1 to 10 . Each source randomly transmits data in bursts of $3.5 \mathrm{~s}$ at intervals of $20 \mathrm{~s}$. Within a burst, each node generates one packet per frame, leading to a packet arrival rate of $4.24 \mathrm{pkt} / \mathrm{s}$. The duration of the advertisement period of ADV-MAC was fixed at $15 \mathrm{~ms}$, which is the optimal value for 5 fresh packets every frame, according to the analytical model in [15]. This value provides an acceptable performance for different numbers of sources. The duration of the advertisement period of ATMA was fixed at $5 \mathrm{~ms}$, which was found to be an adequate value for different numbers of sources. The length of the frame reservation, $n$, is set to 16 frames.

From Fig. 2(a), 2(b) and 2(c), we can see that ATMA requires the minimum energy consumption per packet among all of the protocols, with the lowest latency and the highest packet delivery ratio (PDR). The PDR is almost 100\%, with latency around $200 \mathrm{~ms}$. From Fig. 2(a), it is seen that ATMA consumes $43-45 \%$ less energy than ADV-MAC, $45-61 \%$ less than T-MAC, $44-75 \%$ less than S-MAC $10 \%$ and $64-70 \%$ less than S-MAC $20 \%$. The reduced energy consumption of ATMA is due to a smaller duration of the advertisement period, which is possible because one ADV-ACK exchange can reserve a slot for $n=16$ consecutive frames. This greatly reduces energy consumption. Thus, it is seen that ATMA can adapt well to varying aggregate loads, providing the minimum energy consumption with high PDR and low latency values for bursty traffic scenarios.

2) Effect of reservation length, single hop scenario: In the second set of simulations, we investigate the effect of reservation length on the performance of ATMA. Only ATMA depends on the reservation length. We consider the same area

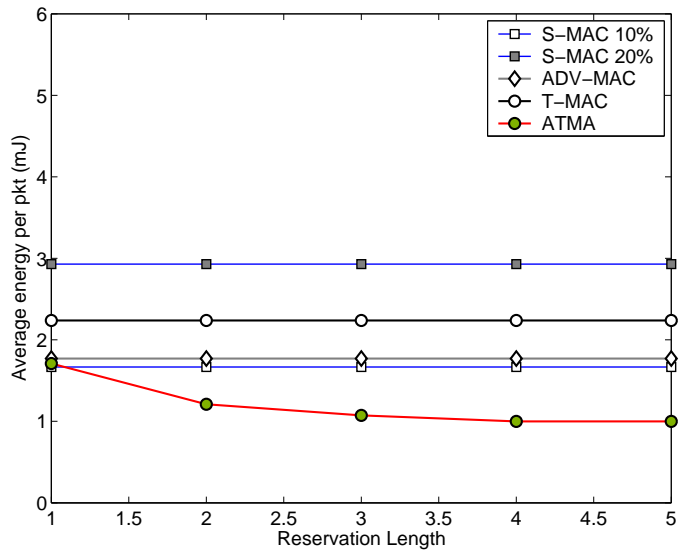

Fig. 3. Single hop scenario. Average energy per packet as a function of data reservation length.

of $50 \mathrm{~m} \times 50 \mathrm{~m}$ with all nodes being in the transmission range of each other, and we set the number of sources to 5 . We vary the reservation length from 1 to 5 frames. As before, each source will randomly transmit data in bursts of $3.5 \mathrm{~s}$ at intervals of $20 \mathrm{~s}$. Within a burst, each node generates one packet per frame. The duration of the advertisement period of ADV-MAC was fixed at $15 \mathrm{~ms}$ as before. The duration of the advertisement period of ATMA was varied from $15 \mathrm{~ms}$ to $5 \mathrm{~ms}$ for acceptable PDR values. We need longer ADV periods for smaller reservation length due to increased contention.

From Fig. 3, we can see that initially, ATMA has the same energy consumption as ADV-MAC. This is because the ADV period duration of both protocols are $15 \mathrm{~ms}$ initially, and a successful ADV/A-ACK exchange only reserves a data slot in that frame. ATMA requires a larger ADV period for reservation length 1 , as it needs to reserve a data slot in each frame for each packet in the burst. This creates high contention overhead. As the reservation length increases, the duration of the ADV period decreases, and the energy consumption decreases. Starting from length 4, the energy consumption per packet becomes constant for ATMA, as a duration of $5 \mathrm{~ms}$ is enough for the ADV period. This constant behavior is observed to continue for higher reservation lengths than 5 frames, which we omit in the figures. The latency and PDR 


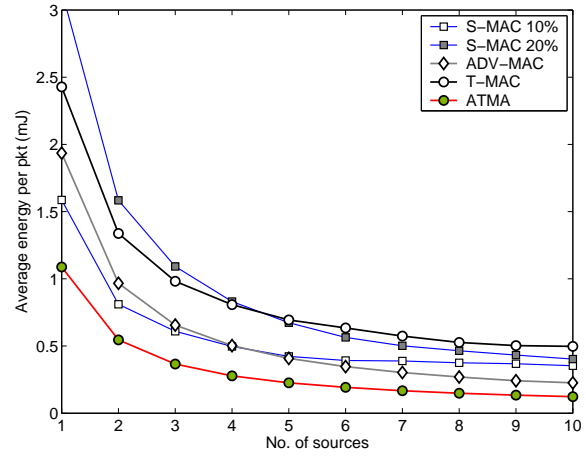

(a) Energy Consumption vs. No. of Sources.

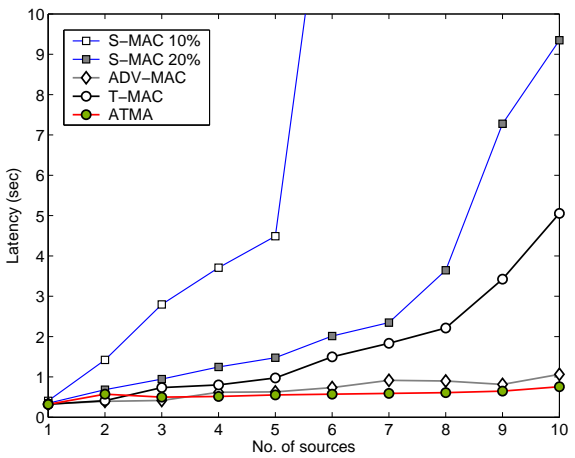

(b) Latency vs. No. of Sources.

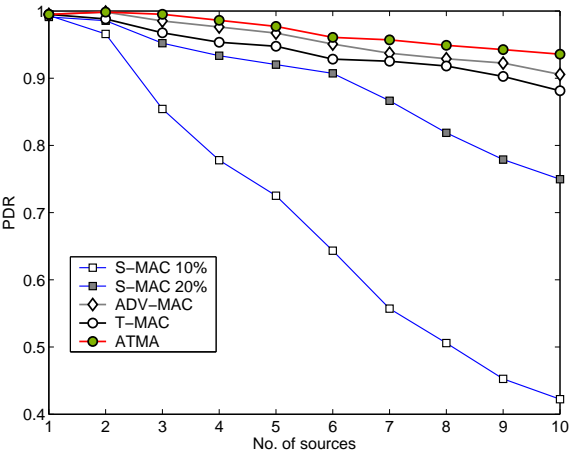

(c) PDR vs. No. of Sources.

Fig. 4. Multi-hop, scenario. Performance as a function of the number of sources for ATMA, ADV-MAC, T-MAC and S-MAC.

of ATMA stays around $200 \mathrm{~ms}$ and $100 \%$, respectively, for all reservation length values investigated and are not shown.

3) Effect of number of sources, multi-hop scenario: In the final set of simulations, we investigate the effect of the number of sources on the performance of the MAC protocols in a multi-hop scenario. We consider 312 nodes in an area of $700 \mathrm{~m}$ $\mathrm{x} 700 \mathrm{~m}$. The sources are deployed uniformly randomly in the area. We vary the average number of sources in the carrier sense range of a node from 1 to 10 . As before, each source randomly transmits data in bursts of $3.5 \mathrm{~s}$ at intervals of $20 \mathrm{~s}$. The duration of the advertisement period of ADV-MAC is fixed at $15 \mathrm{~ms}$ whereas the duration of the same for ATMA is fixed at $5 \mathrm{~ms}$. The length of the frame reservation is set to 16 frames.

The energy consumption, latency and PDR results of the protocols are shown in Fig. 4(a), 4(b) and 4(c), respectively. As seen in the figures, ATMA requires the minimum energy consumption per packet among all of the protocols, while achieving the lowest latency and the highest PDR at the same time. The PDR remains above $90 \%$ with latency below $800 \mathrm{~ms}$. From Fig. 4(a), it is seen that ATMA consumes $43-45 \%$ less energy than ADV-MAC, $55-75 \%$ less than T-MAC, $31-65 \%$ less than S-MAC $10 \%$ and $65-69 \%$ less than S-MAC $20 \%$. Thus, ATMA can adapt to varying loads quite well in multihop scenarios, providing the minimum energy consumption with a high PDR and low latency.

\section{CONCLusion ANd Future Work}

This paper presents ATMA, a new TDMA-based MAC protocol for wireless sensor networks. ATMA uses advertisement and reservation to minimize the energy lost due to idle listening while nodes wait for contention. Detailed simulations show that the proposed protocol adapts nicely to bursty traffic, providing further reductions in energy compared to well-known WSN MAC protocols, Sensor-MAC (S-MAC), Timeout-MAC (T-MAC) as well as a CSMA-based protocol shown to present improvements on these protocols, namely ADV-MAC. An important achievement of ATMA is that it provides these significant energy savings without sacrificing the PDR or latency performances.
In the future, we plan to develop an analytical model for the PDR and energy consumption of ATMA and to optimize the length of the ADV period of ATMA. We also plan to compare ATMA with other TDMA protocols and introduce an energy efficient broadcasting scheme.

\section{REFERENCES}

[1] "IEEE Standard 802.11, Wireless LAN Medium Access Control (MAC) and Physical Layer (PHY) Specifications," LAN MAN Standards Committee of the IEEE Computer Society., June 1999.

[2] "Crossbow Technology Inc." [Online]. Available: http://www.xbow.com/

[3] "Sentilla Corporation." [Online]. Available: http://www.sentilla.com/

[4] W. Ye, J. Heidemann, and D. Estrin, "An energy-efficient mac protocol for wireless sensor networks," in Proceedings of the IEEE Infocom. New York, NY, USA: IEEE, June 2002, pp. 1567-1576.

[5] T. van Dam and K. Langendoen, "An adaptive energy-efficient MAC protocol for wireless sensor networks," in ACM SenSys '03, 2003, pp. $171-180$.

[6] S. Ray, I. Demirkol, and W. Heinzelman, "ADV-MAC: Advertisementbased mac protocol for wireless sensor networks," in The Fifth International Conference on Mobile Ad-hoc and Sensor Networks, Wu Yi Mountain, China, December 2009.

[7] V. Rajendran, K. Obraczka, and J. J. Garcia-Luna-Aceves, "Energyefficient collision-free medium access control for wireless sensor networks," in SenSys '03: Proceedings of the 1st international conference on Embedded networked sensor systems. New York, NY, USA: ACM, 2003, pp. 181-192.

[8] L. v. Hoesel and P. Havinga, "A lightweight medium access protocol (LMAC) for wireless sensor networks," in 1st International Workshop on Networked Sensing Systems, INSS' 04, Tokyo, Japan, Jun. 2004.

[9] L. Bao and J. Garcia-Luna-Aceves, "A new approach to channel access scheduling for ad hoc networks," in In Proc. ACM Seventh Annual International Conference on Mobile Computing and networking, 2001, pp. 210-221.

[10] S. Ramanathan, "A unified framework and algorithm for (T/F/C)DMA channel assignment in wireless networks," Proceedings of the IEEE Infocom '97, vol. 2, pp. 900-907 vol.2, Apr 1997.

[11] S. S. Kulkarni and M. U. Arumugam, "TDMA service for sensor networks," in ICDCSW '04: Proceedings of the 24th International Conference on Distributed Computing Systems Workshops - W7: EC (ICDCSW'04). Washington, DC, USA: IEEE Computer Society, 2004, pp. 604-609.

[12] G. Pei and C. Chien, "Low power TDMA in large wireless sensor networks," in IEEE Military Communications Conference, MILCOM'01., vol. 1, 2001, pp. $347-351$.

[13] B. Tavli and W. R. Heinzelman, "MH-TRACE: multihop time reservation using adaptive control for energy efficiency," IEEE Journal on Selected Areas in Communications, vol. 22, no. 5, pp. 942-953, 2004.

[14] "The Network Simulator NS-2," http://www.isi.edu/nsnam/ns/.

[15] S. Ray, I. Demirkol, and W. Heinzelman, "ADV-MAC: Analysis and optimization of energy efficiency through advertisements for wireless sensor networks," in publication, Elsevier Ad Hoc Networks. 